\title{
Note on Texts
}

When citing, I use square brackets [ ] to indicate expansions of contractions, abbreviations, superscript letters, and tildes. I have not altered the original punctuation in early modern printed or manuscript sources. In most cases, I have kept the original spelling, replacing early modern "u," "i," and "vv" with "v," “j," and "w." Thorns are rendered "th" and yoghs "y." Early modern long esses $(s)$ have also been silently modernized. 
This page intentionally left blank 